\title{
COULOMB SCREENING EFFECT ON THE NUCLEAR-PASTA STRUCTURE
}

\author{
TOSHIKI MARUYAMA ${ }^{1}$, TOSHITAKA TATSUMI ${ }^{2}$, \\ DMITRI N. VOSKRESENSKY ${ }^{3}$, TOMONORI TANIGAWA ${ }^{4,1}$, \\ SATOSHI CHIBA $^{1}$, TOMOYUKI MARUYAMA ${ }^{5}$ \\ 1 Advanced Science Research Center, Japan Atomic Energy Research Institute, \\ Tokai, Ibaraki 319-1195, Japan \\ 2 Department of Physics, Kyoto University, Kyoto 606-8502, Japan \\ 3 Moscow Institute for Physics and Engineering, Kashirskoe sh. 31, Moscow \\ 115409, Russia \\ 4 Japan Society for the Promotion of Science, Tokyo 102-8471, Japan \\ 5 BRS, Nihon University, Fujisawa, Kanagawa 252-8510, Japan
}

\begin{abstract}
Using the density functional theory (DFT) with the relativistic mean field (RMF) model, we study the non-uniform state of nuclear matter, "nuclear pasta". We self-consistently include the Coulomb interaction together with other interactions. It is found that the Coulomb screening effect is significant for each pasta structure but not for the bulk equation of state (EOS) of the nuclear pasta phase.
\end{abstract}

\section{Introduction}

One of the most interesting features of low-density nuclear matter is the possibility of the existence of non-uniform structures, called "nuclear pastas". ${ }^{1}$ At low densities, nuclei in matter are expected to form the Coulomb lattice embedded in the neutron-electron seas, so as to minimize the Coulomb interaction energy. On the other hand, another possibility has been discussed: the stable nuclear shape may change from sphere to cylinder, slab, cylindrical hole, and to spherical hole with increase of the matter density, and "pastas" are eventually dissolved into uniform matter at a certain nucleon density close to the saturation density, $\rho_{s} \simeq 0.16 \mathrm{fm}^{-3}$. The existence 
of such "pasta" phases, instead of the ordinary crystalline lattice of nuclei, would modify several important processes in supernova explosions by changing the hydrodynamic properties and the neutrino opacity in the supernova matter. Also expected is the influence of the "pasta" phases on star quakes of neutron stars and pulsar glitches via the change of mechanical properties of the crust matter.

Several authors have investigated the low-density nuclear matter using various models. ${ }^{1-8}$ Roughly speaking, the favorable nuclear shape is determined by a balance between the surface and the Coulomb energies, as has been shown by previous studies, where the rearrangement effect on the density profile of the charged particles, especially electrons, by the Coulomb interaction is discarded. However, the proper treatment of the Coulomb interaction should be very important, as it is demonstrated in Ref. 9; the screening of the Coulomb interaction by the charged particles may give a large effect on the stability of the geometrical structures.

We have been recently exploring the effect of the Coulomb screening in the context of the structured mixed phases in various first order phase transitions such as hadron-quark deconfinement transition, kaon condensation and liquid-gas transition in nuclear matter. We treat the nuclear "pasta" phases as a part of our project, since they can be considered as structured mixed phases during the liquid-gas transition in nuclear matter.

Our aim here is to study the nuclear "pasta" structures by means of a mean field model, which includes the Coulomb interaction in a proper way, and we figure out how the Coulomb screening effect modifies the previous results without it.

\section{Density Functional Theory with the Relativistic Mean-field Model}

To study the non-uniform nuclear matter, we follow the density functional theory (DFT) with the relativistic mean field (RMF) model. ${ }^{10}$ The RMF model with fields of mesons and baryons is rather simple for numerical calculations, but realistic enough to reproduce main nuclear matter properties. In our framework, the Coulomb interaction is properly included in the equations of motion for nucleons, electrons and the meson mean fields, and we solve the Poisson equation for the Coulomb potential $V_{\text {Coul }}$ selfconsistently with them. Thus the baryon and electron density profiles, as well as the meson mean fields, are determined in a way fully consistent with the Coulomb potential. Note that our framework can be easily extended to 
other situations. For example, if we take into account meson condensations, which are likely realized in a high-density region, we should only add the relevant meson field terms. In Ref. 11 we have included the kaon degree of freedom in such a treatment to discuss kaon condensation in high density regime.

To begin with, we present the thermodynamic potential of the form,

$$
\begin{aligned}
\Omega & =\Omega_{B}+\Omega_{M}+\Omega_{e} \\
\Omega_{B} & =\int d^{3} r\left[\sum_{i=p, n}\left(\frac{2}{(2 \pi)^{3}} \int_{0}^{k_{F i}} d^{3} k \sqrt{m_{B}^{* 2}+k^{2}}-\rho_{i} \nu_{i}\right)\right] \\
\Omega_{M} & =\int d^{3} r\left[\frac{(\nabla \sigma)^{2}+m_{\sigma}^{2} \sigma^{2}}{2}+U(\sigma)-\frac{\left(\nabla \omega_{0}\right)^{2}+m_{\omega}^{2} \omega_{0}^{2}}{2}-\frac{\left(\nabla \rho_{0}\right)^{2}+m_{\rho}^{2} \rho_{0}^{2}}{2}\right], \\
\Omega_{e} & =\int d^{3} r\left[-\frac{1}{8 \pi e^{2}}\left(\nabla V_{\text {Coul }}\right)^{2}-\frac{\left(V_{\text {Coul }}-\mu_{e}\right)^{4}}{12 \pi^{2}}\right]
\end{aligned}
$$

where $\nu_{p}=\mu_{B}-\mu_{e}+V_{\text {Coul }}-g_{\omega N} \omega_{0}-g_{\rho N} \rho_{0}, \quad \nu_{n}=\mu_{B}-g_{\omega N} \omega_{0}+$ $g_{\rho N} \rho_{0}, \quad m_{B}^{*}=m_{B}-g_{\sigma N} \sigma, \quad U(\sigma)=\frac{1}{3} b m_{B}\left(g_{\sigma N} \sigma\right)^{3}+\frac{1}{4} c\left(g_{\sigma N} \sigma\right)^{4}$. Here we used the local-density approximation for nucleons and electrons, while one still should carefully check its validity. The introduction of the density variable is meaningful, if the typical length of the nucleon density variation inside the structure is larger than the inter-nucleon distance, which we assume to be fulfilled. We must also keep in mind that the approximation is broken down for small structure sizes, since quantum effects become prominent there. For the sake of simplicity we also omitted nucleon and electron density derivative terms. If the nucleon length scale were shorter than lengths of changes of the meson mean fields, one could not introduce the derivatives of the nucleon density but could simplify the problem introducing the corresponding contribution to the surface tension. In the given case (when we suppressed the derivative terms mentioned above) the resulting nucleon density follows the changes of the meson mean fields. However, even in this case the presence of the derivative terms (of the same order as for other fields) could affect the numerical results. Here we consider large-size pasta structures and simply discard the density variation effect, as a first-step calculation, while it can be incorporated in the quasi classical way by the derivative expansion within the density functional theory. ${ }^{10}$ The parameters are set to reproduce the nuclear-matter saturation properties. From the variational principle, $\frac{\delta \Omega}{\delta \phi_{i}(\mathbf{r})}=0\left(\phi_{i}=\sigma, \rho_{0}, \omega_{0}, V_{\text {Coul }}\right)$ or 
$\frac{\delta \Omega}{\delta \rho_{i}(\mathbf{r})}=0(i=n, p, e)$, we get the coupled equations of motion as

$$
\begin{aligned}
-\nabla^{2} \sigma+m_{\sigma}^{2} \sigma & =-\frac{d U}{d \sigma}+g_{\sigma N}\left(\rho_{n}^{(s)}+\rho_{p}^{(s)}\right) \\
-\nabla^{2} \omega_{0}+m_{\omega}^{2} \omega_{0} & =g_{\omega N}\left(\rho_{p}+\rho_{n}\right) \\
-\nabla^{2} \rho_{0}+m_{\rho}^{2} \rho_{0} & =g_{\rho N}\left(\rho_{p}-\rho_{n}\right) \\
\nabla^{2} V_{\mathrm{Coul}} & =4 \pi e^{2} \rho_{\mathrm{ch}} \quad\left(\text { charge density } \rho_{\mathrm{ch}}=\rho_{p}+\rho_{e}\right) \\
\mu_{n}=\mu_{B} & =\sqrt{k_{F n}^{2}+m_{B}^{* 2}}+g_{\omega N} \omega_{0}-g_{\rho N} \rho_{0} \\
\mu_{p}=\mu_{B}-\mu_{e} & =\sqrt{k_{F p}^{2}+m_{B}^{* 2}}+g_{\omega N} \omega_{0}+g_{\rho N} \rho_{0}-V_{\mathrm{Coul}} \\
\rho_{e} & =-\left(\mu_{e}-V_{\mathrm{Coul}}\right)^{3} / 3 \pi^{2} .
\end{aligned}
$$

Note that first, the Poisson equation (8) is a highly nonlinear equation for $V_{\text {Coul }}$, since $\rho_{\text {ch }}$ in r.h.s. includes it in a complicated way, and secondly, the Coulomb potential always enters the equation through the gauge invariant combination, $\mu_{e}-V_{\text {Coul }}$.

To solve the above coupled equations numerically, we use the WignerSeitz cell approximation: the space is divided into equivalent cells with some geometrical symmetry. The shape of the cell changes: sphere in three dimensional (3D) calculation, cylinder in 2D and slab in 1D, respectively. Each cell is charge-neutral and all the physical quantities in a cell are smoothly connected to those of the neighbor cell with zero gradients at the boundary. The cell is divided into grid points $\left(N_{\text {grid }} \approx 100\right)$ and the differential equations for fields are solved by the relaxation method with constraints of given baryon number and charge neutrality.

\section{Bulk Property of Finite Nuclei}

Before applying our framework to the problem of the pasta phases in nuclear matter, we check how it can describe finite nuclei. In this calculation, the electron density is set to be zero and the boundary condition or the chargeneutrality condition is not imposed. However, we assumed the spherical shape of nuclei. In Fig. 1 (left panel) we show the density profiles of some typical nuclei. We can see how well our framework may describe the density profiles of these nuclei. To get a better fit, especially around the surface region, we might need to include the derivative terms, as we have mentioned. Fine structures seen in the empirical density profiles, which come from the shell effects (see, e.g., a proton density dip at the center of a light ${ }^{16} \mathrm{O}$ nucleus), cannot be reproduced by the mean field approach. By imposing the 
beta equilibrium, the most stable proton ratio can be obtained for a given mass number. Figure 1 (right panel) shows the mass-number dependence of the binding energy per nucleon and the proton ratio. We can see that the bulk properties of finite nuclei (density, binding energy and proton ratio) are satisfactorily reproduced for our present purpose.

Note that we should adjust a slightly smaller value of the sigma mass than that one usually uses, i.e. $400 \mathrm{MeV}$, to get such a good fit. If we used the popular value of $m_{\sigma} \approx 500 \mathrm{MeV}$, finite nuclei would be overbound by about $3 \mathrm{MeV}$ per nucleon. Although the actual value of the sigma mass (or the omega mass) has little relevance for infinite nuclear matter, it is important for finite nuclei and other non-uniform nucleon systems, since the meson mass characterizes the interaction range and consequently affects, e.g., the nuclear surface property.
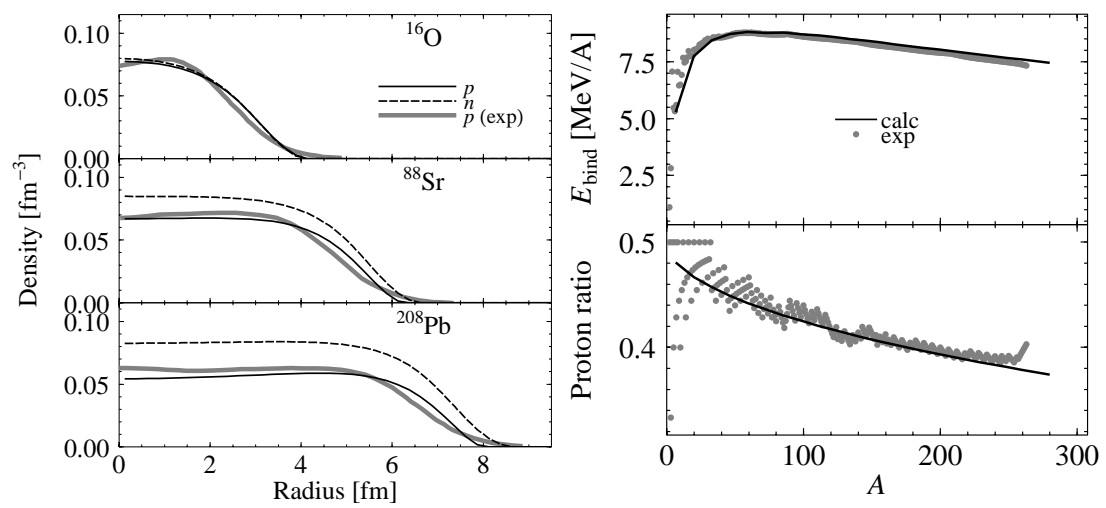

Figure 1. Left: the density profiles of typical nuclei. The proton densities (solid curves) are compared with the experiment. Right: the binding energy per nucleon and the proton ratio of finite nuclei.

\section{Nuclear "Pasta" at Sub-nuclear Densities}

In the density region, where nuclei are about to dissolve into uniform nuclear matter, it is expected that the energetically favorable mixed phase, which consists of nuclei and free nucleons, possesses interesting geometrical structures, such as rod-like and slab-like nuclei and rod-like and spherical bubbles, etc. These nuclei with exotic geometrical shapes are referred to as nuclear "pastas". Phenomenologically the existence of the "pasta" phases instead of the crystalline lattice of nuclei would affect the mechanism of 
supernova explosions and the glitch phenomena in pulsars. Due to these important consequences the "pasta" structure has been studied by number of authors. ${ }^{1-8}$ It is widely accepted that the "pasta" structure is realized basically due to the balance of the Coulomb energy and the surface tension. However, the electron density has been always treated as an uniform background in the standard treatments. Here we study the nuclear "pasta" structure within our framework, which consistently treats the Coulomb potential and the electron distribution.

\subsection{Symmetric Nuclear Matter and the Coulomb Screening Effect}

First, we focus on symmetric nuclear matter (relevant to supernova matter at the initial stage of collapse) where the Coulomb screening effect by electrons is expected to be large. Figure 2 shows some typical density profiles in the Wigner-Seitz cells. The geometrical dimension of the cell is denoted as "3D" (three dimensional), etc. The horizontal axis in each panel denotes the radial distance from the center of the cell, and the boundary is indicated by the hatch. The nuclear "pasta" structures are clearly seen. Note that the electron density profile becomes no more uniform due to the Coulomb screening.

The phase diagram of the matter structure is shown in Fig. 3 (left). The size of the cell $R_{\text {cell }}$ is optimized with precision of $1 \mathrm{fm}$, and the lowest energy configurations are chosen among various geometrical structures. In the figure, there never appears the spherical-hole configuration. This is one of the consequences of the Coulomb screening effect. It should be noted that appearance of the pasta structures is also sensitive to the choice of the effective interaction, as discussed by Oyamatsu et al. ${ }^{12}$

To elucidate the Coulomb screening effect, there are two possible ways: one is to compare our results with those given by solving the equations of motion for fields and the density profiles neglecting the Coulomb potential $V_{\text {Coul }}$. Then the Coulomb energy is calculated by the use of the density profiles thus determined and finally added to the total energy, as in the simple bulk calculations. The optimum cell size is determined by this total energy including the Coulomb energy. The other way is to compare our results with those given by only discarding $V_{\text {Coul }}$ in r.h.s. of the Poisson equation, while keeping it in other equations of motion. It is equivalent to the assumption of the uniform electron density distribution, which has been used in the previous studies; ${ }^{3-8}$ there protons interact with each other 


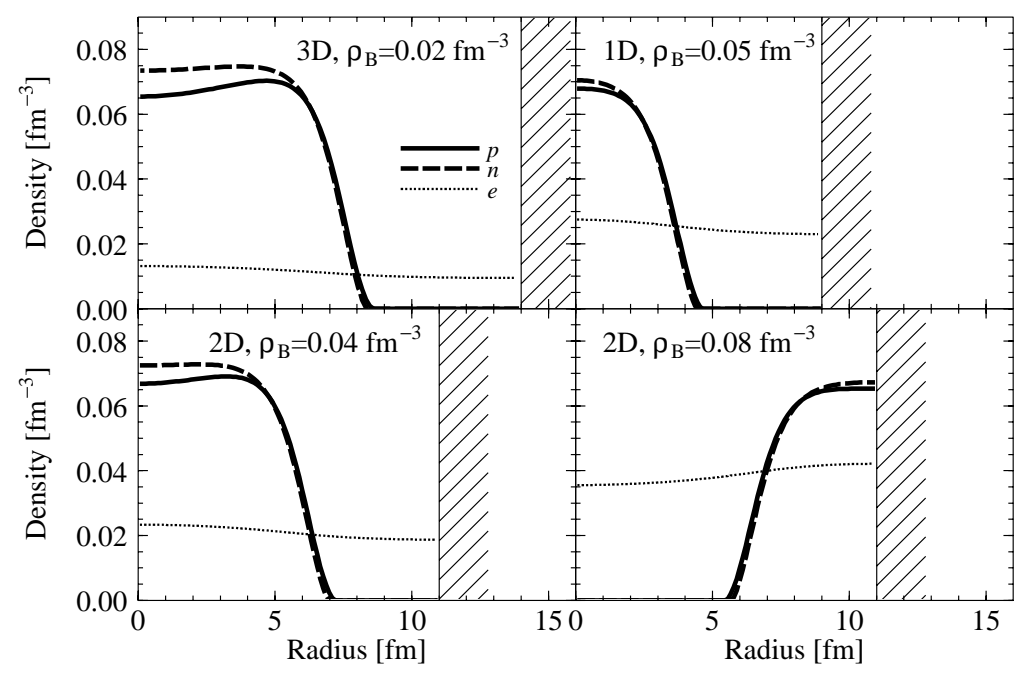

Figure 2. Examples of the density profiles in the cell for symmetric nuclear matter (droplet, rod, slab, and tube).

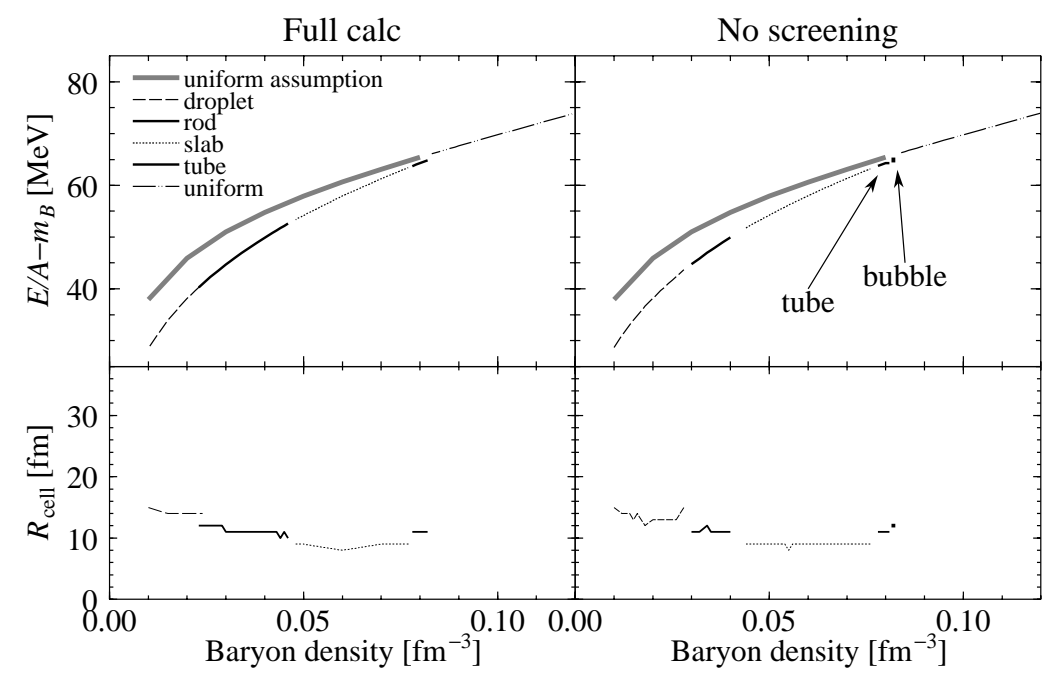

Figure 3. Left: the binding energy per nucleon and the cell size of symmetric nuclear matter. Right: the same as the left panel with the uniform electron distribution. 
and may form a non-uniform structure through the balance between the nuclear surface tension and the Coulomb interaction in a uniform electron background. Thus the density rearrangement effect is partially taken into account for protons, while it is completely neglected for electrons. The first way may be standard to extract the Coulomb effect and to compare our full calculation with the bulk one; actually this way has been taken in the context of kaon condensation ${ }^{11}$ or hadron-quark deconfinement transition. ${ }^{9}$ However, we don't take the first way and dare to take the second way here to compare our results with the previous ones given by the uniform-electron calculation.

We show in the right panel of Fig. 3 the results without the Coulomb screening (uniform-electron calculation). The region of each structure (droplet, rod, etc.) is different from that given by the full calculation. Especially, the "bubble" (spherical hole) appears in this case. Since the appearance of various geometrical structures and their region depend on the very subtle energy difference, the Coulomb screening has a significant influence on the sequence of the different pasta phases.

Comparing the case of uniform matter with the case of the pasta phases, one can see that the non-uniform structures reduce the energy. However, the Coulomb screening effect on the bulk EOS (difference between left and right panels of Fig. 3) is rather small.

\subsection{Nuclear Matter in Beta Equilibrium}

Next, we discuss the nuclear matter in beta equilibrium, which is relevant to stable neutron stars. Figure 4 (left) shows the density profiles at several densities. Only the case for three dimensional (3D) geometrical structure is shown, since $2 \mathrm{D}$ and $1 \mathrm{D}$ cases are energetically unfavored in our calculation. One can see the proton-enriched droplets embedded into the neutron sea at low densities. The EOS with the $3 \mathrm{D}$ phases is shown in the right panel of Fig. 4. In the beta equilibrium nuclear matter the effect of the non-uniform structure becomes much less compared to that for symmetric nuclear matter. Since the electron fraction is small, the Coulomb screening effect should not be remarkable.

\section{Summary and Concluding Remarks}

We have discussed the low-density nuclear matter structures "nuclear pastas" and elucidated the Coulomb screening effect. Using a self-consistent framework based on DFT and RMF, we took into account the Coulomb 

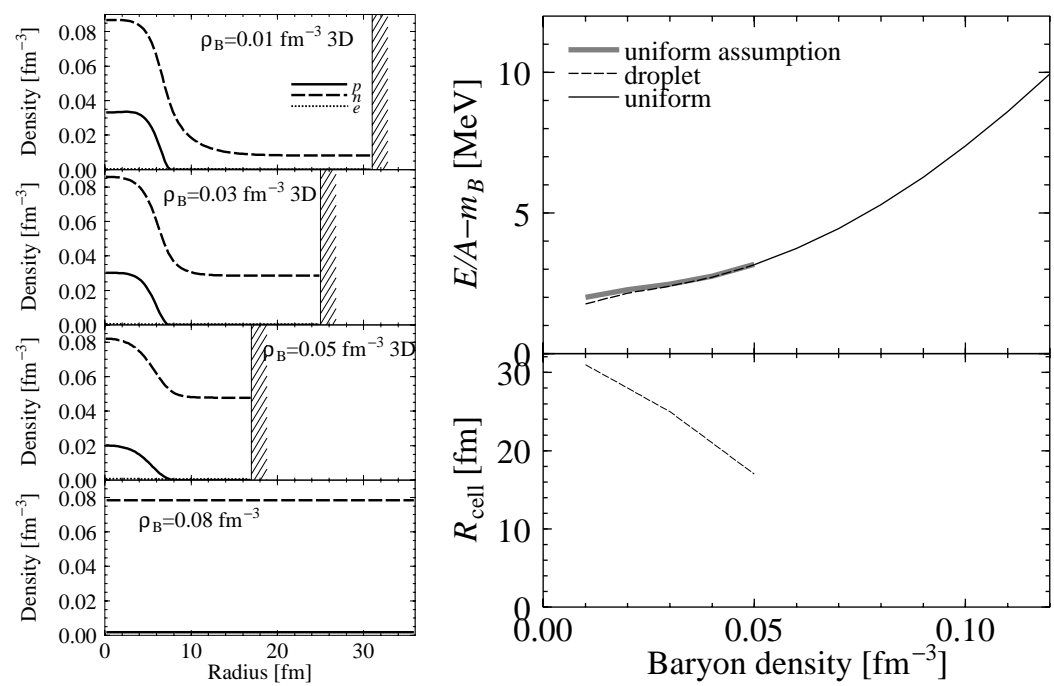

Figure 4. Left: examples of the density profiles of nuclear matter in beta equilibrium. The electron density is too small to see clearly. Right: the binding energy per nucleon and the cell size.

interaction in a proper way and numerically solved the coupled equations of motion to extract the density profiles of nucleons.

First we have checked how realistic our framework is by calculating the bulk properties of finite nuclei as well as the saturation properties of nuclear matter, and found it can describe both features satisfactorily.

In symmetric nuclear matter, we have observed the "nuclear pasta" structures with various geometries at sub-nuclear densities. The appearance of the pasta structures significantly lowers the energy, i.e. softens the EOS, while the energy differences between various geometrical structures are rather small. So the Coulomb screening effect and the rearrangement of the charge density can affect such changes of geometrical structures in spite of that its absolute value is rather small in comparison with nuclear interaction energy.

By comparing the results with and without the Coulomb screening, we have seen that the self-consistent inclusion of the Coulomb interaction changes the phase diagram. In particular one of the pasta configurations appears only when the Coulomb screening is switched off in our calculation. The effects of the Coulomb screening on the EOS, on the other hand, was found to be small. 
We have also studied the structure of nuclear matter in beta equilibrium. There we have observed only proton-enriched droplet in the neutron sea. No other geometrical structures like rod, slab, etc. appeared.

Detailed discussions about the Coulomb screening effect on the nuclear pasta phases will be reported elsewhere.

\section{References}

1. D. G. Ravenhall, C. J. Pethick and J. R. Wilson, Phys. Rev. Lett. 27, 2066 (1983).

2. M. Hashimoto, H. Seki and M. Yamada, Prog. Theor. Phys. 71, 320 (1984).

3. R. D. Williams and S. E. Koonin, Nucl. Phys. A435, 844 (1985).

4. K. Oyamatsu, Nucl. Phys. A561, 431 (1993).

5. C. P. Lorentz, D. G. Ravenhall and C. J. Pethick, Phys. Rev. Lett. 25, 379 (1993).

6. K. S. Cheng, C. C. Yao and Z. G. Dai, Phys. Rev. C55, 2092 (1997).

7. T. Maruyama, K. Niita, K. Oyamatsu, T. Maruyama, S. Chiba and A. Iwamoto, Phys. Rev. C57, 655 (1998). T. Kido, T. Maruyama, K. Niita and S. Chiba, Nucl. Phys. A663-664, 877 (2000).

8. G. Watanabe, K. Iida and K. Sato, Nucl. Phys. A676, 445 (2000); G. Watanabe, K. Sato, K. Yasuoka and T. Ebisuzaki, Phys. Rev. C66, 012801 (2002).

9. D.N. Voskresensky, M. Yasuhira and T. Tatsumi, Phys. Lett. B541, 93 (2002); Nucl. Phys. A723, 291 (2003); T. Tatsumi and D. N. Voskresensky, this proceedings (nucl-th/0312114).

10. Density Functional Theory, ed. E. K. U. Gross and R. M. Dreizler, Plenum Press (1995).

11. T. Maruyama et al., this proceedings.

12. K. Oyamatsu et al., this proceedings. 